\title{
Fenología de algunas especies que son alimento para la pava aliblanca Penélope albipennis
}

\author{
Phenology of some species that are food for the White-winged Guan \\ Penelope albipennis
}

Facultad de Ciencias Biológicas, Universidad Nacional Pedro Ruiz Gallo, Ciudad Universitaria, Juan XXIII No 391, Lambayeque, Perú. Delgado: guidelg2001@yahoo.es

\section{Joaquín R. Martos, Mariella Scarpati, Consuelo Rojas y Guillermo E. Delgado}

\begin{abstract}
Resumen
En la Reserva Ecológica Privada Chaparrí, Chongoyape (Lambayeque) se evaluó la fenología (desarrollo vegetativo, floración y fructificación) de 17 especies vegetales que alimentan a la pava aliblanca (Penélope albipennis Taczanowski). Las evaluaciones fueron mensuales entre julio 2004 y junio 2005, en tres zonas denominadas: bosque seco de planicie, bosque seco de quebrada húmeda y bosque seco de ladera. La temperatura tuvo correlación con el desarrollo vegetativo, floración y fructificación. De las 17 especies evaluadas, 6 especies $(35,3 \%)$ estuvieron disponibles como alimento de la pava aliblanca durante todo el año, en tanto que de las 11 especies restantes $(64,7 \%)$ fueron de carácter estacional; durante todo el año la pava aliblanca dispone de oferta alimenticia de alguna de las especies evaluadas.
\end{abstract}

Palabras claves: Desarrollo vegetativo, floración, fructificación, bosque seco ecuatorial, pava aliblanca

\section{Abstract}

The phenological traits (vegetative growth, flowering and fruiting) of 17 plant species that feed the White-winged Guan (Penelope albipennis Taczanowski) was assessed in the Ecological Reserve of Chaparrí, Chongoyape (Lambayeque). The evaluations were monthly between July 2004 and June 2005 in three areas known as: plain dry forest, dry forest to wet and dry forest ravine slope. The temperature was correlated with vegetative growth, flowering and fruiting. Of the 17 species tested, 6 species $(35,3 \%)$ were available as food for White-winged Guan throughout the year, while the remaining 11 species $(64,7 \%)$ were seasonal, year-round the White-winged Guan available food supply of the species evaluated.

\section{Introducción}

La pava aliblanca (Penelope albipennis Taczanowski), perteneciente a la familia Cracidae, es una ave endémica del norte del Perú, considerada extinta en el siglo XIX y redescubierta en la quebrada de San Isidro, Lambayeque, en 1977 (Díaz 1998). Las principales amenazas de la pava aliblanca son la persecución directa por el hombre y la destrucción de su hábitat por extractores de madera y colonizadores de nuevas tierras de cultivo. Varios esfuerzos para su protección y conservación se han realizado en determinadas áreas naturales y en condición de semicautiverio. Una de estas áreas la constituye la Reserva Ecológica Privada (REP) Chaparrí, Chongoyape, Lambayeque.

La REP Chaparrí forma parte del ecosistema del bosque seco ecuatorial, un bioma muy frágil que se encuentra sólo en el sur del Ecuador y en el norte del Perú, que no obstante contener muchas especies endémicas está en constante amenaza ante la pérdida de su composición original (Brack \& Mendiola 2000). En efecto, Sagástegui et al. (1999) han indicado que la región norte y nororiente del Perú, presenta cerca del 26\% de especies vegetales endémicas, algo similar ha sido señalado en la diversidad y endemismo de vertebrados (Aguilar, 1994), como es el caso de la pava aliblanca.

Como fuente de alimento de la pava aliblanca se ha mencionado a Prosopis chilensis (algarrobo), Acacia macracantha (faique) y Scutia spicata (lipe) (De Macedo 1979) y Muntingia calabura (cerezo), Pithecellobium excellsum (chaquiro), Ficus passiflora (higuerón), Loxopterigium huasango (hualtaco), Cordia lutea (overo), Bursera graveolens (palo santo), Carica parviflora (papaya silvestre) y Capparis avicennifolia (vichayo) (Lerner 2003); sin embargo, en ninguno de estos trabajos se abordan aspectos fenológicos de las especies mencionadas. El entendimiento de la fenología (ciclo de vida de las plantas, desarrollo vegetativo, floración y fructificación) relacionada con el clima, resulta crítico en el manejo y sostenibilidad del ecosistema (Croat 1969), además, de ser base de estudios sobre polinización, dispersión, fisiológicos y taxonómicos (Alencar 1994).

Por otro lado, a diferencia de lo que ocurre con la flora de la Amazonía donde se han realizado varios trabajos fenológicos (Gautier \& Spichiger 1986; Villasana \& Suárez 1997; Ruiz, 2000; Zárate et al. 2006; entre otros), pocos son los trabajos de este tema realizados en el bosque seco ecuatorial. Entre estos trabajos tenemos el realizado por Silva \& Higaona (1985) que estudiaron la fenología del algarrobo, faique y overo, en las Pampas de Olmos (Lambayeque), Borchert (1995) quién realizó un amplio estudio sobre la fenología y periodicidad en la floración en las especies neotropicales del bosque seco ecuatorial, Bridgewater et al. (2003) que realizaron un análisis florístico y fitogeográfico preliminar de la flora leñosa del bosque seco en el norte del Perú y Leal-Pinedo \& Linares-Palomino (2005) quienes analizaron y discutieron los patrones de estructura y diversidad de los bosques secos estacionales de la Reserva de Biosfera del Noroeste, Parque Nacional Cerros de Amotape y Zona Reservada de Tumbes, en el Perú.

En este trabajo presentamos los resultados de la evaluación fenológica -desarrollo vegetativo, floración y fructificación- de algunas especies que alimentan a Penelope albipennis (pava aliblanca) en la REP Chaparrí, Chongoyape, Lambayeque, lo cual, facilitará la planificación de la crianza de esta importante ave endémica de la región.

\section{Material y métodos}

Las evaluaciones se realizaron una vez por mes desde julio 2004 a junio 2005. 


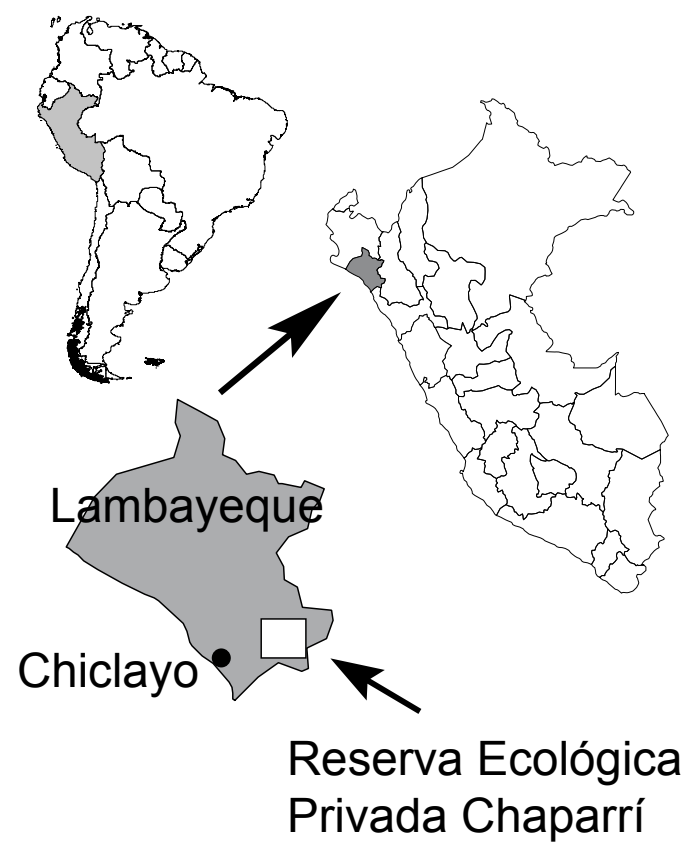

Figura 1. Ubicación del área de estudio $06^{\circ} 31^{\prime} 17^{\prime \prime} S-79^{\circ} 27^{\prime} 33^{\prime \prime} \mathrm{W}$ y Reserva Ecológica Privada Chaparrí, Chongoyape, Lambayeque, Perú.

\section{Área de estudio}

El área de estudio estuvo localizada entre los 06 31'17"S y 79²7'33"W (329625,733 m Este y 721100,827 m Norte, huso 17 , hemisferio M), y corresponde a la quebrada Pavas, en las faldas del cerro Pavas, abarca el bosque seco adyacente y al cerro Chaparrí, al pie del que se encuentra la quebrada Hualcatal. Esta área de estudio se encuentra ubicada en la Reserva Ecológica Privada de Chaparrí, la cual tiene una extensión de 34312 hectáreas y abarca los terrenos de la comunidad campesina Santa Catalina de Chongoyape, ocupando territorios del distrito de Chongoyape, provincia de Chiclayo, región Lambayeque y distritos de Llama y Miracosta, provincia de Chota, región Cajamarca (Fig. 1).

\section{Zonas de muestreo y vegetación}

El presente trabajo se realizó en tres zonas de muestreo (Fig. 1, tabla 1), las que presentaban las siguientes características descritas brevemente:

Bosque seco de planicie, se encuentra en suelo arenoarcilloso y pedregoso, topografía plana a ligeramente inclinada con pequeñas ondulaciones, dependiendo de las lluvias más no de las aguas subterráneas. Presenta el menor número de especies muestreadas, que alimentan a la pava aliblanca, aunque el número de individuos muestreados fue ligeramente superior al del bosque seco de ladera; sin embargo, la densidad en individuos es muy rala. Entre las especies representativas tenemos a Grabowskia boerhaaviaefolia (Solanaceae), Cucumis dipsaceus (Cucurbitaceae), Luffa operculata (Cucurbitaceae), Passiflora foetida (Passifloraceae), Vallesia glabra (Apocynaceae), Macroptilium atropurpureum (Papilionaceae), Ipomoea purpurea (Convolvulaceae) y Eragrostis cilianensis (Poaceae).

Bosque seco de quebrada húmeda, se caracteriza por la presencia de la quebrada Pavas, con suelo arcilloso y rocas de grandes dimensiones, una fuerte inclinación del terreno y ocurrencia de agua durante todo el año aunque en caudal variable, siendo mayor en los meses de verano (enero - marzo) debido a las precipitaciones pluviales estacionales. Presenta el mayor número de especies muestreadas, que alimentan a la pava aliblanca, y casi duplica el número de individuos muestreados; el sotobosque es ligeramente denso e irregularmente abierto. Entre las especies representativas tenemos a Piper aduncum (Piperaceae), Baccharis salicifolia (Asteraceae), Cestrum auriculatum, (Solanaceae), Solanum americanum (Solanaceae), Peperomia dolabriformis (Piperaceae), Tessaria integrifolia (Asteraceae) y Lycopersicon pimpinellifollium (Solanaceae).

Bosque seco de ladera, se encuentra en suelo areno-arcilloso y pedregoso, con fuerte inclinación topográfica y al igual que el bosque seco de planicie depende de las lluvias más no de las aguas subterráneas. Presenta un número de especies e individuos muestreados de manera similar a lo que ocurre en el bosque seco

Tabla 1. Relación de individuos evaluados en el bosque seco de planicie, bosque seco de quebrada húmeda y bosque seco de ladera en la REP Chaparrí, Chongoyape (Lambayeque-Perú) ${ }^{1}$.

\begin{tabular}{|c|c|c|c|c|c|}
\hline & \multicolumn{2}{|l|}{ Especie } & $\begin{array}{l}\text { Bosque seco de } \\
\text { planicie }\end{array}$ & $\begin{array}{c}\text { Bosque seco de } \\
\text { quebrada }\end{array}$ & $\begin{array}{c}\text { Bosque seco de } \\
\text { ladera }\end{array}$ \\
\hline & Nombre científico / Familia & Nombre vulgar & \multicolumn{3}{|c|}{$\mathrm{N} .{ }^{\circ}$ individuos } \\
\hline 1 & Acacia macracantha/ Mimosaceae & Faique, huarango & 2 & 2 & 2 \\
\hline 2 & Acnistus arborescens/ Solanaceae & Tuple & & 6 & \\
\hline 3 & Bursera graveolens/ Burseraceae & Palo santo & 2 & 2 & 2 \\
\hline 4 & Cesalpinia paipai/Caesalpiniaceae & Pai pai & 2 & & 2 \\
\hline 5 & Capparis avicennifolia/ Capparaceae & Vichayo & 2 & 2 & 2 \\
\hline 6 & Capparis scabrida/ Capparaceae & Sapote & 4 & & 2 \\
\hline 7 & Carica parviflora/ Caricaceae & Papayo silvestre & & 2 & 2 \\
\hline 8 & Cercidium praecox/ Caesalpiniaceae & Palo verde, canaquil & 3 & & \\
\hline 9 & Cordia lutea/ Boraginaceae & Overo & 2 & 2 & 2 \\
\hline 10 & Eriotheca ruizii/ Bombacaceae & Pasallo & 2 & 2 & 2 \\
\hline 11 & Ficus nymphaeifolia/ Moraceae & Higuerón & & 6 & \\
\hline 12 & Loxopterigium huasango/Anacardiaceae & Hualtaco & 2 & 2 & 2 \\
\hline 13 & Muntingia calabura/ Elaeocarpaceae & Cerecillo & & 6 & \\
\hline 14 & Pithecellobium excellsum/ Mimosaceae & Chaquiro & & 2 & 2 \\
\hline 15 & Phithecolobium multiflorum/ Mimosaceae & Angolo & & 2 & 1 \\
\hline 16 & Prosopis pallida/Mimosaceae & Algarrobo & 3 & 2 & \\
\hline 17 & Scutia spicata/Rhamnaceae & Lipe & & 5 & \\
\hline
\end{tabular}

1 Bosque seco de planicie: Coordenada 17M 670602 - $670935 \mathrm{mE}, 9278026$ - $9278398 \mathrm{mN}$ y altitud (m) 468 - 520; Bosque seco de quebrada húmeda: Coordenada $17 \mathrm{M} 670282$ - 670892 mE, 9278446 - 9278926 mN y altitud (m) 447 - 592 y Bosque seco de ladera: Coordenada 17 M669822 - 670116 mE, 9279180 - 9279414 mN y altitud (m) 688 - 727. 


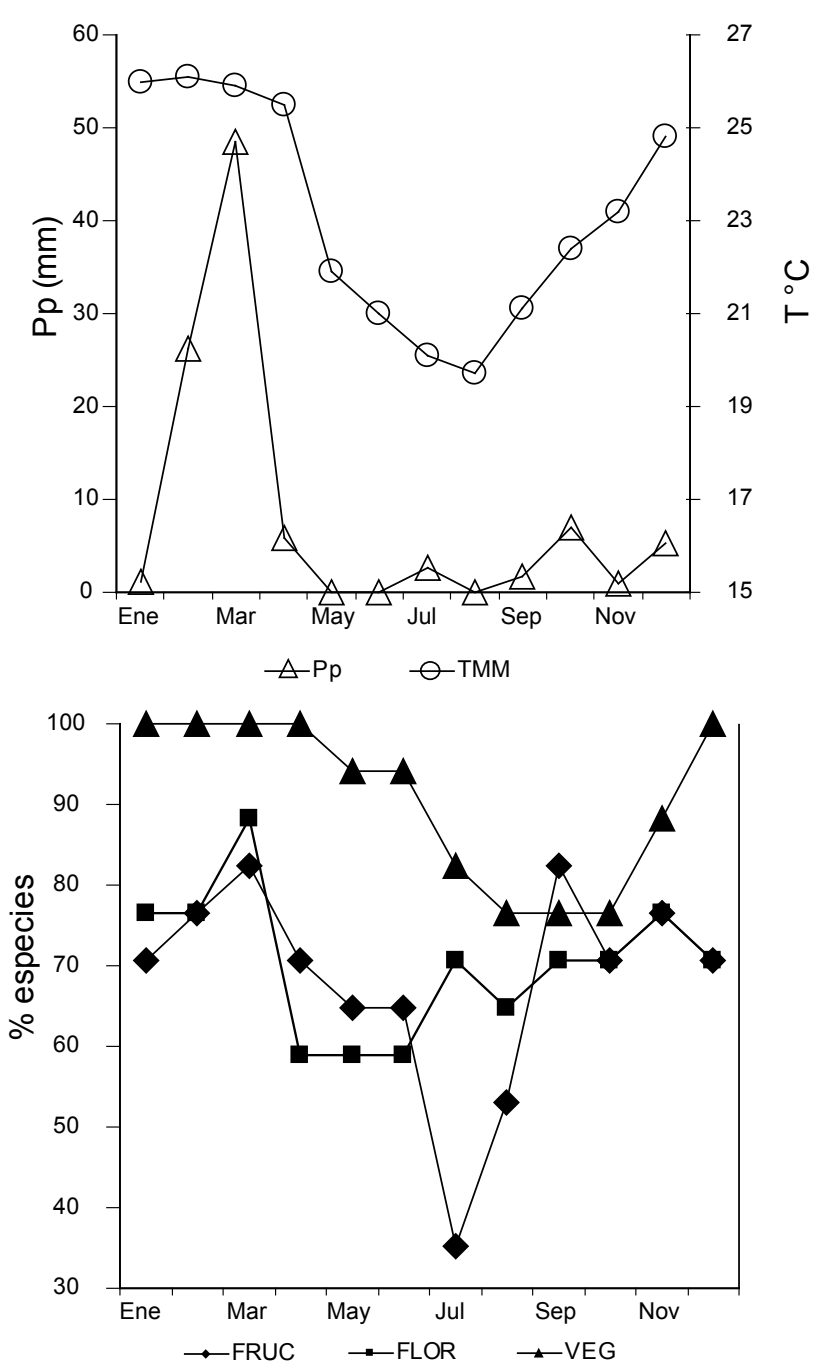

Figura 2. Relación entre la precipitación media $(\mathrm{Pp})$ y la temperatura (TMM) sobre el desarrollo vegetativo (VEG), la floración (FLOR) y fructificación (FRUC), entre julio 2004 a junio 2005, en la Reserva Ecológica Privada Chaparrí, Chongoyape, Lambayeque.

de planicie; sin embargo, su densidad en individuos es muy alta. Entre las especies representativas tenemos a Alternanthera pubiflora (Amaranthaceae), Wedelia latifolia (Asteraceae), Bougainvillea pachyphylla (Nyctaginaceae) y Trichocereus sp. (Cactaceae).

Selección de especímenes en las zonas de muestreo. Fueron consideradas un total de 17 especies, que por observaciones previas y durante el estudio, referencias bibliográficas (De Macedo 1979; Lerner 2003) y consultas con los pobladores del lugar, alimentan a la pava aliblanca. De cada especie se seleccionó 2 individuos por zona de muestreo; sin embargo, algunas especies estuvieron ausentes en una y hasta en dos zonas de muestreo, razón por la cual se incrementó el número de individuos en las otras zonas de muestreo hasta alcanzar el número máximo de 6 individuos por especie (Tabla 1). Cada individuo fue marcado y determinado su posición mediante GPS (Global Position Satelital)

Clima. La información climatológica de temperatura y precipitación fue proporcionada por la Estación Tinajones (0639'2,6”S, 79²5'22,5”W y 218 m de altitud), ubicada en el distrito de Chongoyape, Provincia de Chiclayo y Departamento de Lambayeque, encontrándose en el ámbito geográfico de la REP Chaparrí. La temperatura media anual (julio 2004 - junio
2005) fue $23,1^{\circ} \mathrm{C}$ variando entre $19,5^{\circ} \mathrm{C}$, media de agosto/2004, y $26,1^{\circ} \mathrm{C}$, media de febrero/2005; la precipitación media anual fue $8,3 \mathrm{~mm}$, oscilando entre $0,0 \mathrm{~mm}$ en mayo y junio/2004 y agosto/2005 y 48,5 mm en marzo/2005 (Figura 2).

Fisiografía y altitud. La REP Chaparrí se extiende a ambas márgenes del río Chancay y está delimitada por cadenas de cerros que determinan la cuenca del río, una zona agrícola central adyacente al río y a las principales quebradas y luego pampas planas, con muy poca pendiente, extendiéndose hasta las faldas de los cerros que rodean la reserva. La altitud va de 150 a $1500 \mathrm{~m}$ (cerro El Alumbral), registrándose las cumbres más altas los cerros Chaparrí (1346 m), Los Bravos (1350 m) y Querguer (1250 m).

Periodos de floración y fructificación. En cada individuo marcado se determinó su altura total, altura comercial, el diámetro a la altura del pecho (DAP) y la cobertura. En la determinación de la altura total (e) se utilizó el hipsómetro de Merrit, teniendo en cuenta la longitud del brazo del operador (b), la altura del árbol (representado en la regla) (h) y la distancia al árbol (d), aplicando la fórmula e $=\mathrm{b} \times \mathrm{h} / \mathrm{d}$.

Se registró el desarrollo vegetativo (ramas con hojas y brotes) y del desarrollo reproductivo (flores y frutos). También fue registrada la presencia de flores y frutos caídos para la verificación de la ocurrencia de ambos procesos. Se consideraron los porcentajes de individuos con respuestas de desarrollo vegetativo o reproductivo. Las plantas en floración y/o fructificación fueron colectadas, herborizadas y depositadas en el Herbario Ruiz Gallo de la Universidad Nacional Pedro Ruiz Gallo de Lambayeque.

\section{Resultados y discusión}

\section{Distribución de especies en las zonas de muestreo}

La terminología utilizada en la clasificación de las zonas de muestreo se ha basado en la clasificación propuesta por Brack \& Mendiola (2000) pero con algunas modificaciones. La zona del bosque seco de planicie sería lo que fue propuesto como "sabana verde en época de lluvia" y la del bosque seco de ladera lo que fue propuesto como "bosque seco". Estos autores no hacen referencia al bosque seco de quebrada húmeda sino al bosque de galería, para referirse al bosque que crece junto a los ríos y cauces secos, dependiendo del agua subterránea, lo cual no fue el caso en el presente estudio.

En la tabla 1 se muestra la distribución de las 17 especies evaluadas en las tres zonas de muestreo estudiadas. En las zonas de muestreo, correspondientes al bosque seco de planicie y bosque seco de ladera, se evaluaron 10 y 11 especies, con 24 y 23 individuos, respectivamente, en tanto que en el bosque seco de quebrada húmeda 14 especies con 43 individuos. Algunas especies como $A$. macracantha Humb. \& Bonpl. ex Willd., $B$. graveolens (H.B.K.) Triana y Planch., C. avicennifolia H.B.K., C. lutea Lam., Eriotheca ruizii y L. huasango ocurren en las tres zonas de muestreo, en tanto que Cesalpinia paipai R. \& P., Capparis scabrida H.B.K., C. parviflora (A. DC.) Solms-Laubach, P. excellsum, Phithecolobium multiflorum (H.B.K.) Benth y Prosopis pallida (H. \& B. ex Willd) H.B.K ocurren en dos zonas de muestreo y Acnistus arborescens, Cercidium praecox (R. \& P.) Harms, Ficus nymphaefolia, M. calabura y S. spicata (H. \& B. ex Schul) Weberb., ocurren en solamente una zona de muestreo, la que correspondió al bosque seco de quebrada húmeda. De estas especies solamente $A$. arborescens, F. nymphaefolia y $M$. calabura, 
Tabla 2. Características dendrológicas de los individuos evaluados en el bosque seco de planicie, bosque seco de quebrada húmeda y bosque de ladera en la REP Chaparrí, Chongoyape (Lambayeque-Perú) 1 .

\begin{tabular}{|c|c|c|c|c|c|}
\hline No & Especie & Altura total (m) & Altura comercial (m) & $\mathrm{DAP}(\mathrm{cm})$ & Cobertura $(\mathrm{m})$ \\
\hline 1 & Acacia macracantha & 4,8 & 1,0 & 17,6 & 3,8 \\
\hline 2 & Acnistus arborescens & 5,1 & - & 5,5 & 5,1 \\
\hline 3 & Bursera graveolens & 6,5 & 1,9 & 20,8 & 4,7 \\
\hline 4 & Cesalpinia paipai & 3,5 & 2,2 & 16,8 & 1,3 \\
\hline 5 & Capparis avicennifolia & 2,2 & - & 5,9 & 2,2 \\
\hline 6 & Capparis scabrida & 4,6 & 1,7 & 20,0 & 2,9 \\
\hline 7 & Carica parviflora & 2,7 & - & 10,6 & 2,7 \\
\hline 8 & Cercidium praecox & 3,3 & 0,5 & 17,9 & 2,8 \\
\hline 9 & Cordia lutea & 2,5 & - & 4,8 & 2,5 \\
\hline 10 & Eriotheca ruizii & 8,4 & 4,0 & 29,3 & 4,4 \\
\hline 11 & Ficus nymphaeifolia & 12,2 & 5,8 & 44,7 & 6,4 \\
\hline 12 & Loxopterigium huasango & 8,5 & 1,9 & 28,7 & 6,6 \\
\hline 13 & Muntingia calabura & 9,1 & 6,2 & 14,9 & 2,9 \\
\hline 14 & Pithecellobium excellsum & 6,1 & 1,0 & 5,6 & 5,1 \\
\hline 15 & Phithecolobium multiflorum & 10,5 & 5,5 & 29,1 & 5,0 \\
\hline 16 & Prosopis pallida & 6,1 & 1,6 & 32,2 & 4,6 \\
\hline 17 & Scutia spicata & 2,9 & - & 3,9 & 2,9 \\
\hline
\end{tabular}

${ }^{1}$ Altura total, Altura que incluye la altura comercial y cobertura; altura comercial, altura del fuste aprovechable del árbol y DAP, diámetro a la altura del pecho.

desarrollan en ambientes de alta humedad como es el caso del bosque seco de quebrada húmeda. Carica parviflora, por su condición herbácea, no desarrolla en el bosque seco de planicie, C. scabrida prefiere el bosque seco de planicie y bosque seco de ladera y no el bosque seco de quebrada húmeda, en tanto que la ausencia de $C$. praecox y $P$. pallida en el bosque seco de ladera obedece más a un factor de depredación que a distribución natural. La presencia de $S$. spicata, únicamente en el bosque de quebrada húmeda y no en el bosque seco de planicie y bosque seco de ladera responde al carácter aleatorio del muestreo y $C$. lutea se comporta como la especie predominante del sotobosque en las tres zonas de muestreo estudiadas.

Es conocido que en los bosques húmedos y lluviosos las diferencias encontradas entre el número de especies e individuos presentes en las zonas de muestreo obedecen a las características ecológicas de los ambientes en que se desarrollan, en función a la demanda edáfica, hidrológica y de humedad, es decir, la estacionalidad climática de la región condiciona la dinámica del bosque (Gentry \& Ortiz, 1993). En nuestro estudio, la humedad permanente del bosque seco de quebrada húmeda condiciona la presencia de determinadas especies que difícilmente prosperarían en una condición ecológica diferente.

\section{Características dendrológicas y fenológicas}

Fueron determinadas las características dendrológicas y fenológicas de 88 individuos correspondientes a 17 especies distribuidas en 12 familias. Estas características se encuentran especificadas en las tablas 2 y 3 .

Acacia macracantha. Árbol mayor de $5 \mathrm{~m}$ de altura, inflorescencia en glomérulos de color amarillo-anaranjado y fruto legumbre marrón-negruzco. Durante todo el año alcanza un frondoso desarrollo vegetativo con un máximo de $84,2 \%$ en marzo, en tanto que la mayor aparición de brotes ocurre entre enero a abril con un máximo de $40 \%$ en enero; la floración se inicia en setiembre extendiéndose hasta junio sin superar el $6 \%$ y la fructificación sigue un ritmo fluctuante con la aparición de frutos verdes y maduros, entre abril a diciembre, con un máximo de $16,7 \%$ de frutos maduros en abril.
Acnistus arborescens. Arbusto de $5 \mathrm{~m}$ de altura, flores blancas y frutos baya amarillos. El desarrollo vegetativo ocurre durante todo el año con un máximo de $80 \%$ entre setiembre a febrero, en tanto que el mayor desarrollo de brotes se observó en enero y agosto con más de $90 \%$; la floración ocurre entre octubre a febrero con un máximo de 63,3\% en noviembre; la fructificación sigue una tendencia similar observándose $86,7 \%$ de frutos verdes en abril y $56 \%$ de frutos maduros en mayo.

Bursera graveolens. Árbol caducifolio de $7-8 \mathrm{~m}$ de altura, flores en racimo y fruto drupa de color verde rojizo. El desarrollo vegetativo abarca de diciembre a junio, alcanzando un máximo de $85 \%$ en febrero, permaneciendo el resto del ańo carente de hojas; los brotes se desarrollan entre diciembre a marzo con un máximo de $50 \%$, también en febrero; la floración sigue la misma tendencia con un máximo de $21,6 \%$ en enero, en tanto que la fructificación ocurre simultáneamente con un máximo de $13,3 \%$ de frutos maduros, también en enero.

Cesalpinia paipai. Árbol de 3-4 m de altura, flores en corimbo y frutos legumbre de color marrón oscuro. Presenta un vigoroso desarrollo vegetativo durante todo el año, predominando entre setiembre a marzo con valores cercanos a $65 \%$; los brotes se desarrollan entre febrero a marzo con valores alrededor de $21 \%$; la floración ocurre de julio a febrero alcanzando un máximo de $20 \%$ en noviembre, mientras que la fructificación es muy errática a lo largo del año, sin embargo, valores entre 5 a $10 \%$, entre frutos verdes y maduros, se han observado entre enero a marzo.

Capparis avicennifolia. Árbol irregular de hasta $3 \mathrm{~m}$ de altura, flores en corimbo de olor agradable y frutos baya, oblongogloboso, verdes y de sabor dulce. El desarrollo vegetativo ocurre durante todo el año con un máximo de $83,3 \%$ en julio, en tanto que entre enero a agosto se observa el mayor desarrollo de brotes con 20 a 40\%; la floración ocurre, también, durante todo el año destacando enero con $42,5 \%$ y la fructificación es mayor entre octubre a marzo destacando febrero con $52,5 \%$, entre frutos verdes y maduros.

Capparis scabrida. Árbol de hasta $5 \mathrm{~m}$ de altura, flores en corimbo y frutos baya, aovado-oblongo. El desarrollo vegetativo es muy vigoroso durante todo el ańo alcanzando valores cercanos 
Tabla 3. Calendario del desarrollo vegetativo (ramas con hojas y brotes), floración (botones y flores) y fructificación (frutos verdes y maduros). Indicando el porcentaje de individuos con respuesta de desarrollo vegetativo, floración y fructificación en la REP Chaparrí, Chongoyape (Lambayeque-Perú).

\begin{tabular}{|c|c|c|c|c|c|c|c|c|c|c|c|c|c|}
\hline \multirow[b]{2}{*}{ Especie } & \multicolumn{12}{|c|}{ Meses del año $\mathbf{o}^{1,2}$} & \multirow[b]{2}{*}{ Respuesta (\%) } \\
\hline & En & $\mathbf{F b}$ & Mz & $\mathrm{Ab}$ & My & $\mathrm{Ju}$ & $\mathrm{Jl}$ & Ag & Se & Oc & $\mathbf{N v}$ & Dc & \\
\hline \multirow{3}{*}{ Acacia macracantha } & $\mathrm{Vg}$ & $\mathrm{Vg}$ & $\mathrm{Vg}$ & $\mathrm{Vg}$ & $\mathrm{Vg}$ & $\mathrm{Vg}$ & $\mathrm{Vg}$ & $\mathrm{Vg}$ & $\mathrm{Vg}$ & $\mathrm{Vg}$ & $\mathrm{Vg}$ & $\mathrm{Vg}$ & $35,0-84,2$ \\
\hline & $\mathrm{Fl}$ & $\mathrm{Fl}$ & $\mathrm{Fl}$ & $\mathrm{Fl}$ & $\mathrm{Fl}$ & $\mathrm{Fl}$ & & & $\mathrm{Fl}$ & $\mathrm{Fl}$ & $\mathrm{Fl}$ & $\mathrm{Fr}$ & $0,1-5,6$ \\
\hline & & & & $\mathrm{Fr}$ & $\mathrm{Fr}$ & $\mathrm{Fr}$ & $\mathrm{Fr}$ & $\mathrm{Fr}$ & $\mathrm{Fr}$ & $\mathrm{Fr}$ & $\mathrm{Fr}$ & & $3,3-16,7$ \\
\hline \multirow{3}{*}{ Acnistus arborescens } & $\mathrm{Vg}$ & $\mathrm{Vg}$ & $\mathrm{Vg}$ & $\mathrm{Vg}$ & $\mathrm{Vg}$ & $\mathrm{Vg}$ & $\mathrm{Vg}$ & $\mathrm{Vg}$ & $\mathrm{Vg}$ & $\mathrm{Vg}$ & $\mathrm{Vg}$ & $\mathrm{Vg}$ & $3,3-91,6$ \\
\hline & $\mathrm{Fl}$ & $\mathrm{Fl}$ & $\mathrm{Fl}$ & $\mathrm{Fl}$ & $\mathrm{Fl}$ & $\mathrm{Fl}$ & $\mathrm{Fl}$ & $\mathrm{Fl}$ & $\mathrm{Fl}$ & $\mathrm{Fl}$ & $\mathrm{Fl}$ & $\mathrm{Fl}$ & $0,8-95,0$ \\
\hline & $\mathrm{Fr}$ & $\mathrm{Fr}$ & $\mathrm{Fr}$ & $\mathrm{Fr}$ & $\mathrm{Fr}$ & $\mathrm{Fr}$ & $\mathrm{Fr}$ & $\mathrm{Fr}$ & $\mathrm{Fr}$ & $\mathrm{Fr}$ & $\mathrm{Fr}$ & $\mathrm{Fr}$ & $0,7-86,7$ \\
\hline \multirow{3}{*}{ Bursera graveolens } & $\mathrm{Vg}$ & $\mathrm{Vg}$ & $\mathrm{Vg}$ & $\mathrm{Vg}$ & $\mathrm{Vg}$ & $\mathrm{Vg}$ & & & & & $\mathrm{Vg}$ & $\mathrm{Vg}$ & $23,3-85,0$ \\
\hline & $\mathrm{Fl}$ & $\mathrm{Fl}$ & $\mathrm{Fl}$ & $\mathrm{Fl}$ & $\mathrm{Fl}$ & $\mathrm{Fl}$ & & & & & $\mathrm{Fl}$ & $\mathrm{Fl}$ & $1,2-45,0$ \\
\hline & $\mathrm{Fr}$ & $\mathrm{Fr}$ & $\mathrm{Fr}$ & $\mathrm{Fr}$ & $\mathrm{Fr}$ & $\mathrm{Fr}$ & & & & & & & $1,6-21,0$ \\
\hline \multirow{3}{*}{ Cesalpinia paipai } & $\mathrm{Vg}$ & $\mathrm{Vg}$ & $\mathrm{Vg}$ & $\mathrm{Vg}$ & $\mathrm{Vg}$ & $\mathrm{Vg}$ & $\mathrm{Vg}$ & $\mathrm{Vg}$ & $\mathrm{Vg}$ & $\mathrm{Vg}$ & $\mathrm{Vg}$ & $\mathrm{Vg}$ & $47,8-65,0$ \\
\hline & $\mathrm{Fl}$ & $\mathrm{Fl}$ & & & & & $\mathrm{Fl}$ & $\mathrm{Fl}$ & & $\mathrm{Fl}$ & $\mathrm{Fl}$ & $\mathrm{Fl}$ & $1,8-21,3$ \\
\hline & $\mathrm{Fr}$ & $\mathrm{Fr}$ & $\mathrm{Fr}$ & & $\mathrm{Fr}$ & $\mathrm{Fr}$ & & $\mathrm{Fr}$ & $\mathrm{Fr}$ & & $\mathrm{Fr}$ & $\mathrm{Fr}$ & $0,5-10,0$ \\
\hline \multirow{3}{*}{ Capparis avicennifolia } & $\operatorname{Vg}$ & $\mathrm{Vg}$ & $\mathrm{Vg}$ & $\mathrm{Vg}$ & $\mathrm{Vg}$ & $\mathrm{Vg}$ & $\mathrm{Vg}$ & $\operatorname{Vg}$ & $\mathrm{Vg}$ & $\mathrm{Vg}$ & $\mathrm{Vg}$ & $\mathrm{Vg}$ & $37,5-83,3$ \\
\hline & $\mathrm{Fl}$ & $\mathrm{Fr}$ & $\mathrm{Fl}$ & $\mathrm{Fl}$ & $\mathrm{Fl}$ & $\mathrm{Fl}$ & $\mathrm{Fl}$ & $\mathrm{Fl}$ & $\mathrm{Fl}$ & $\mathrm{Fl}$ & Fl & $\mathrm{Fl}$ & $1,6-42,5$ \\
\hline & $\mathrm{Fr}$ & $\mathrm{Fr}$ & $\mathrm{Fr}$ & $\mathrm{Fr}$ & & $\mathrm{Fr}$ & & $\mathrm{Fr}$ & $\mathrm{Fr}$ & $\mathrm{Fr}$ & $\mathrm{Fr}$ & $\mathrm{Fr}$ & $1,7-36,7$ \\
\hline \multirow{3}{*}{ Capparis scabrida } & $\mathrm{Vg}$ & $\mathrm{Vg}$ & $\mathrm{Vg}$ & $\mathrm{Vg}$ & $\mathrm{Vg}$ & $\mathrm{Vg}$ & $\mathrm{Vg}$ & $\mathrm{Vg}$ & $\mathrm{Vg}$ & $\mathrm{Vg}$ & $\mathrm{Vg}$ & $\mathrm{Vg}$ & $68,7-88,5$ \\
\hline & $\mathrm{Fl}$ & & $\mathrm{Fl}$ & $\mathrm{Fl}$ & $\mathrm{Fl}$ & $\mathrm{Fl}$ & $\mathrm{Fl}$ & $\mathrm{Fl}$ & Fl & $\mathrm{Fl}$ & Fl & $\mathrm{Fl}$ & $0,3-56,4$ \\
\hline & $\mathrm{Fr}$ & $\mathrm{Fr}$ & $\mathrm{Fr}$ & $\mathrm{Fr}$ & & & & & $\mathrm{Fr}$ & $\mathrm{Fr}$ & $\mathrm{Fr}$ & $\mathrm{Fr}$ & $2,0-26,9$ \\
\hline \multirow{3}{*}{ Carica parviflora } & $\mathrm{Vg}$ & $\mathrm{Vg}$ & $\mathrm{Vg}$ & $\mathrm{Vg}$ & & & & & & & & $\mathrm{Vg}$ & $10,0-58,8$ \\
\hline & $\mathrm{Fl}$ & $\mathrm{Fl}$ & $\mathrm{Fl}$ & $\mathrm{Fl}$ & $\mathrm{Fl}$ & $\mathrm{Fl}$ & & & & $\mathrm{Fl}$ & $\mathrm{Fl}$ & $\mathrm{Fl}$ & $2,5-62,5$ \\
\hline & $\mathrm{Fr}$ & $\mathrm{Fr}$ & $\mathrm{Fr}$ & $\mathrm{Fr}$ & $\mathrm{Fr}$ & $\mathrm{Fr}$ & & & & & $\mathrm{Fr}$ & & $0,5-22,5$ \\
\hline \multirow{3}{*}{ Cercidium praecox } & $\mathrm{Vg}$ & $\mathrm{Vg}$ & $\mathrm{Vg}$ & $\mathrm{Vg}$ & $\mathrm{Vg}$ & $\mathrm{Vg}$ & $\mathrm{Vg}$ & $\mathrm{Vg}$ & $\mathrm{Vg}$ & $\mathrm{Vg}$ & $\mathrm{Vg}$ & $\mathrm{Vg}$ & $0,3-95,0$ \\
\hline & & & & & & & $\mathrm{Fl}$ & $\mathrm{Fl}$ & $\mathrm{Fl}$ & & & & $2,0-6,6$ \\
\hline & $\mathrm{Fr}$ & & $\mathrm{Fr}$ & & & & & & $\mathrm{Fr}$ & $\mathrm{Fr}$ & $\mathrm{Fr}$ & $\mathrm{Fr}$ & $2,3-43,3$ \\
\hline \multirow{3}{*}{ Cordia lutea } & $\mathrm{Vg}$ & $\mathrm{Vg}$ & $\mathrm{Vg}$ & $\mathrm{Vg}$ & $\mathrm{Vg}$ & $\mathrm{Vg}$ & $\mathrm{Vg}$ & $\mathrm{Vg}$ & $\mathrm{Vg}$ & $\mathrm{Vg}$ & $\mathrm{Vg}$ & $\mathrm{Vg}$ & $3,3-58,8$ \\
\hline & $\mathrm{Fl}$ & $\mathrm{Fl}$ & $\mathrm{Fl}$ & $\mathrm{Fl}$ & $\mathrm{Fl}$ & $\mathrm{Fl}$ & $\mathrm{Fl}$ & $\mathrm{Fl}$ & $\mathrm{Fl}$ & $\mathrm{Fl}$ & $\mathrm{Fl}$ & $\mathrm{Fl}$ & $3,3-21,6$ \\
\hline & & $\mathrm{Fr}$ & $\mathrm{Fr}$ & $\mathrm{Fr}$ & $\mathrm{Fr}$ & $\mathrm{Fr}$ & & $\mathrm{Fr}$ & $\mathrm{Fr}$ & $\mathrm{Fr}$ & $\mathrm{Fr}$ & $\mathrm{Fr}$ & $0,9-4,6$ \\
\hline \multirow{3}{*}{ Eriotheca ruizii } & $\mathrm{Vg}$ & $\mathrm{Vg}$ & $\mathrm{Vg}$ & $\mathrm{Vg}$ & $\mathrm{Vg}$ & $\mathrm{Vg}$ & & & & & $\mathrm{Vg}$ & $\mathrm{Vg}$ & $0,2-79,2$ \\
\hline & & $\mathrm{Fl}$ & $\mathrm{Fl}$ & $\mathrm{Fl}$ & $\mathrm{Fl}$ & $\mathrm{Fl}$ & $\mathrm{Fl}$ & & & & & & $3,3-37,7$ \\
\hline & & & & & & $\mathrm{Fr}$ & $\mathrm{Fr}$ & $\mathrm{Fr}$ & $\mathrm{Fr}$ & $\mathrm{Fr}$ & $\mathrm{Fr}$ & $\mathrm{Fr}$ & $2,0-25,0$ \\
\hline \multirow{3}{*}{ Ficus nymphaeifolia } & $\mathrm{Vg}$ & $\mathrm{Vg}$ & $\mathrm{Vg}$ & $\mathrm{Vg}$ & $\mathrm{Vg}$ & $\mathrm{Vg}$ & $\mathrm{Vg}$ & $\mathrm{Vg}$ & $\mathrm{Vg}$ & $\mathrm{Vg}$ & $\mathrm{Vg}$ & $\mathrm{Vg}$ & $2,1-84,0$ \\
\hline & & & $\mathrm{Fl}$ & $\mathrm{Fl}$ & & & $\mathrm{Fl}$ & $\mathrm{Fl}$ & $\mathrm{Fl}$ & $\mathrm{Fl}$ & Fl & $\mathrm{Fl}$ & $2,9-15,8$ \\
\hline & $\mathrm{Fr}$ & $\mathrm{Fr}$ & & & $\mathrm{Fr}$ & & $\mathrm{Fr}$ & $\mathrm{Fr}$ & $\mathrm{Fr}$ & $\mathrm{Fr}$ & $\mathrm{Fr}$ & $\mathrm{Fr}$ & $0,3-17,1$ \\
\hline \multirow{3}{*}{ Loxopterigium huasango } & $\mathrm{Vg}$ & $\mathrm{Vg}$ & $\mathrm{Vg}$ & $\mathrm{Vg}$ & $\mathrm{Vg}$ & $\mathrm{Vg}$ & $\mathrm{Vg}$ & & & & & $\mathrm{Vg}$ & $4,2-82,8$ \\
\hline & $\mathrm{Fl}$ & $\mathrm{Fl}$ & $\mathrm{Fl}$ & & & $\mathrm{Fl}$ & & & & & & & $2,5-34,2$ \\
\hline & & & $\mathrm{Fr}$ & $\mathrm{Fr}$ & $\mathrm{Fr}$ & $\mathrm{Fr}$ & & & & & & & $2,1-8,0$ \\
\hline \multirow{3}{*}{ Muntingia calabura } & $\mathrm{Vg}$ & $\mathrm{Vg}$ & $\mathrm{Vg}$ & $\mathrm{Vg}$ & $\mathrm{Vg}$ & $\mathrm{Vg}$ & $\mathrm{Vg}$ & $\mathrm{Vg}$ & $\mathrm{Vg}$ & $\mathrm{Vg}$ & $\mathrm{Vg}$ & $\mathrm{Vg}$ & $2,1-100,0$ \\
\hline & $\mathrm{Fl}$ & $\mathrm{Fl}$ & $\mathrm{Fl}$ & & & & $\mathrm{Fl}$ & $\mathrm{Fl}$ & $\mathrm{Fl}$ & $\mathrm{Fl}$ & $\mathrm{Fl}$ & $\mathrm{Fl}$ & $0,9-15,0$ \\
\hline & $\mathrm{Fr}$ & $\mathrm{Fr}$ & $\mathrm{Fr}$ & $\mathrm{Fr}$ & $\mathrm{Fr}$ & $\mathrm{Fr}$ & $\mathrm{Fr}$ & & $\mathrm{Fr}$ & $\mathrm{Fr}$ & $\mathrm{Fr}$ & $\mathrm{Fr}$ & $0,3-13,8$ \\
\hline & $\mathrm{Vg}$ & $\mathrm{Vg}$ & $\mathrm{Vg}$ & $\mathrm{Vg}$ & $\mathrm{Vg}$ & $\mathrm{Vg}$ & $\mathrm{Vg}$ & $\mathrm{Vg}$ & $\mathrm{Vg}$ & $\mathrm{Vg}$ & $\mathrm{Vg}$ & $\mathrm{Vg}$ & $12,5-88,8$ \\
\hline Pithecellobium excellsum & $\mathrm{Fl}$ & $\mathrm{Fl}$ & $\mathrm{Fl}$ & & $\mathrm{Fl}$ & & $\mathrm{Fl}$ & $\mathrm{Fl}$ & $\mathrm{Fl}$ & $\mathrm{Fl}$ & $\mathrm{Fl}$ & $\mathrm{Fl}$ & $4,0-47,5$ \\
\hline & & $\mathrm{Fr}$ & $\mathrm{Fr}$ & $\mathrm{Fr}$ & $\mathrm{Fr}$ & & & $\mathrm{Fr}$ & $\mathrm{Fr}$ & $\mathrm{Fr}$ & $\mathrm{Fr}$ & $\mathrm{Fr}$ & $0,5-32,5$ \\
\hline & $\mathrm{Vg}$ & $\mathrm{Vg}$ & $\mathrm{Vg}$ & $\mathrm{Vg}$ & $\mathrm{Vg}$ & $\mathrm{Vg}$ & $\mathrm{Vg}$ & $\mathrm{Vg}$ & $\mathrm{Vg}$ & $\mathrm{Vg}$ & $\mathrm{Vg}$ & $\mathrm{Vg}$ & $50,0-85,0$ \\
\hline Phithecolobium multiflorum & & $\mathrm{Fl}$ & $\mathrm{Fl}$ & $\mathrm{Fl}$ & $\mathrm{Fl}$ & $\mathrm{Fl}$ & $\mathrm{Fl}$ & $\mathrm{Fl}$ & $\mathrm{Fl}$ & $\mathrm{Fl}$ & $\mathrm{Fl}$ & & $0,6-15,0$ \\
\hline & $\mathrm{Fr}$ & $\mathrm{Fr}$ & $\mathrm{Fr}$ & $\mathrm{Fr}$ & $\mathrm{Fr}$ & $\mathrm{Fr}$ & $\mathrm{Fr}$ & $\mathrm{Fr}$ & $\mathrm{Fr}$ & $\mathrm{Fr}$ & & & $0,4-41,4$ \\
\hline & $\mathrm{Vg}$ & $\mathrm{Vg}$ & $\mathrm{Vg}$ & $\mathrm{Vg}$ & $\mathrm{Vg}$ & $\mathrm{Vg}$ & $\mathrm{Vg}$ & $\mathrm{Vg}$ & $\mathrm{Vg}$ & $\mathrm{Vg}$ & $\mathrm{Vg}$ & $\mathrm{Vg}$ & $54,2-79,2$ \\
\hline Prosopis pallida & $\mathrm{Fl}$ & & $\mathrm{Fl}$ & & & & $\mathrm{Fl}$ & & Fl & $\mathrm{Fl}$ & $\mathrm{Fl}$ & $\mathrm{Fl}$ & $5,0-39,2$ \\
\hline & $\mathrm{Fr}$ & $\mathrm{Fr}$ & $\mathrm{Fr}$ & $\mathrm{Fr}$ & & & & & $\mathrm{Fr}$ & $\mathrm{Fr}$ & $\mathrm{Fr}$ & $\mathrm{Fr}$ & $0,3-56,7$ \\
\hline & $\mathrm{Vg}$ & $\mathrm{Vg}$ & $\mathrm{Vg}$ & $\mathrm{Vg}$ & $\mathrm{Vg}$ & $\mathrm{Vg}$ & $\mathrm{Vg}$ & $\mathrm{Vg}$ & $\mathrm{Vg}$ & $\mathrm{Vg}$ & $\mathrm{Vg}$ & $\mathrm{Vg}$ & $20,0-71,0$ \\
\hline Scutia spicata & $\mathrm{Fl}$ & $\mathrm{Fl}$ & $\mathrm{Fl}$ & & & & & $\mathrm{Fl}$ & $\mathrm{Fl}$ & & & & $0,8-50,0$ \\
\hline & $\mathrm{Fr}$ & $\mathrm{Fr}$ & $\mathrm{Fr}$ & & & & & & $\mathrm{Fr}$ & & & & $0,8-21,0$ \\
\hline
\end{tabular}

1 Meses del año: julio 2004-junio 2005

$2 \mathrm{Vg}$, desarrollo vegetativo; Fl, floración y Fr, fructificación.

a $90 \%$, no obstante, la iniciación de brotes es mayor en febrero y junio con un valor promedio de $30 \%$; la floración predomina entre marzo y abril con $30 \%$ y entre julio a setiembre con $55 \%$, en promedio; la fructificación abarca de setiembre a abril, con un máximo de $30 \%$ en enero, entre frutos verdes y maduros.
Carica parviflora. Especie dioica, de tronco herbáceo que alcanza 2-3 m de altura, flores vistosas y frutos baya de pulpa amarillo-anaranjado. El desarrollo vegetativo abarca de diciembre a abril con un máximo cercano a $80 \%$, meses donde también ocurre la formación de brotes; la floración se inicia en setiembre 
y la fructificación en enero, prolongándose, ambos eventos, hasta junio, alcanzando los valores más altos entre enero a marzo con 80 y $23 \%$, respectivamente.

Cercidium praecox. Árbol de $3 \mathrm{~m}$ de altura, flores en racimos y frutos legumbre. El desarrollo vegetativo ocurre durante todo el año alcanzando sus mayores valores entre enero a marzo con un máximo de $95 \%$, en tanto que la formación de brotes abarca de diciembre a marzo con un máximo de $43,3 \%$ en enero; la floración ocurre entre julio a setiembre y la fructificación entre setiembre a enero con un máximo de $56,6 \%$ en octubre, entre frutos verdes y maduros.

Cordia lutea. Arbusto caducifolio de 2,5 m de altura, flores amarillas en panícula y frutos baya blanquecinos y globosos. Exhibe un profuso desarrollo vegetativo durante todo el ańo destacando febrero a abril con valores cercanos a $60 \%$, mientras que los brotes se desarrollan entre enero a junio con un máximo de $20 \%$ en enero y febrero; la floración fue mayor entre diciembre a junio, con $20 \%$ en promedio y la fructificación ocurre a lo largo del año con un máximo de $14 \%$ en agosto, entre frutos verdes y maduros.

Eriotheca ruizii. Árbol dominante de $8-9 \mathrm{~m}$ de altura, caducifolio, flores en panícula de color marfil y fruto cápsula con semillas lanuginosas. El desarrollo vegetativo abarca de noviembre a junio con un máximo de 79,2\% en febrero, en tanto la inducción de brotes fue mayor en enero con 33,5\%; la floración se inicia en marzo y concluye en julio con un máximo de $37,7 \%$ en junio y la fructificación ocurre entre julio a diciembre con un máximo de $25 \%$ en agosto.

Ficus nymphaeifolia. Árbol dominante con una altura superior a $12 \mathrm{~m}$ y fruto sicono, globoso y sésil. El desarrollo vegetativo ocurre durante todo el año con un máximo de $84 \%$ en enero, mientras que la mayor formación de brotes ocurre en julio con $33,5 \%$; la floración es baja durante el año con un máximo de $15,8 \%$ en marzo y la fructificación, entre frutos verdes y maduros fue mayor en los meses de setiembre y octubre con 16,8 y $17,1 \%$, respectivamente.

Loxopterigium huasango. Árbol de $8 \mathrm{~m}$ de altura, caducifolio, flores pequeñas y fruto sámara. El desarrollo vegetativo ocurre entre enero a julio con un máximo de $82,8 \%$ en enero, en tanto que los brotes alcanzan su mayor formación en diciembre con $40 \%$; la floración ocurre entre enero a marzo con un máximo de $17,5 \%$ en enero y la fructificación ocurre entre marzo a mayo con $10,9 \%$ en abril, entre frutos verdes y maduros.

Muntingia calabura. Árbol de $10 \mathrm{~m}$ de altura, flores blancas y fruto baya, globoso, de color rojo. El desarrollo vegetativo ocurre durante todo el año alcanzando un máximo de $100 \%$ en enero, en tanto que la formación de brotes se verifica únicamente en los meses de verano, con un máximo de $30,8 \%$ en febrero; la floración abarca de julio a febrero con un máximo de 15\% en agosto, mientras que la formación de frutos se verifica casi todo el ańo, excepto los meses de julio y agosto, con un máximo de $19,6 \%$ en noviembre, entre frutos verdes y maduros.

Pithecellobium excellsum. Arbusto de $6 \mathrm{~m}$ de altura a más, flores blancas, frutos legumbre de color rojo y espiralados y semillas negras, brillosas, con arilo rojo o blanco. El desarrollo vegetativo ocurre durante todo el ańo con un máximo de $88,8 \%$ en julio, en tanto que la aparición de brotes únicamente se observó en febrero con $12,5 \%$; la floración siguió un proceso errático durante todo el año con un máximo de $47,5 \%$ en agosto, mientras que la formación de frutos verdes y maduros fue mayor en noviembre con $36 \%$.

Phithecolobium multiflorum. Árbol de $10 \mathrm{~m}$ de altura y fruto legumbre de color marrón rojizo brillante. El desarrollo vegetativo es frondoso y ocurre durante todo el año con un máximo de $85 \%$, en tanto que la aparición de brotes ocurre entre enero a marzo; la floración es observada durante todo el año, excepto los meses de enero y diciembre, con un máximo de $15 \%$ en abril, mientras que la ocurrencia de frutos sigue una tendencia similar con un máximo de $41,4 \%$ de frutos verdes en enero y $23,6 \%$ de frutos maduros en abril.

Prosopis pallida. Arbol de $8 \mathrm{~m}$ de altura, con flores en espiga y fruto legumbre, la algarroba, que tiene un mesocarpo pastoso y dulce. Presenta un uniforme desarrollo vegetativo durante todo el ańo con un máximo de $80 \%$ y $20 \%$ de brotes entre enero a marzo; la floración ocurre desde setiembre a marzo coincidiendo con la aparición de los frutos, verdes durante todo ese periodo con un máximo de $56,7 \%$ en noviembre y maduros con un máximo de $30 \%$, entre febrero y marzo.

Scutia spicata. Arbusto extendido, con espinas prominentes y fruto drupáceo de color rojo vinoso. Aunque el desarrollo vegetativo ocurre durante todo el año es mayor entre setiembre a febrero alcanzando un máximo de $70 \%$ en ramas con hojas y $80 \%$ de brotes en julio; la floración fue muy pobre, observándose un máximo de $75 \%$ entre agosto a setiembre; estos periodos de floración coinciden con los de fructificación con un máximo de $26 \%$ en setiembre.

El desarrollo vegetativo ocurre durante todo el año en la mayoría de las especies evaluadas $(76,4 \%)$ con excepción de 4 especies (23,6\%), B. graveolens, C. parviflora, E. ruizii y $L$. huasango, carentes de desarrollo vegetativo entre los meses de mayo a noviembre, donde precisamente se registra la menor precipitación pluvial; en general, el desarrollo vegetativo abarcó un periodo de 5 a 12 meses con una media de 10,9 meses. La floración, en tanto, ocurre durante todo el año únicamente en 3 especies (17,6\%), A. arborescens, $C$. avicennifolia y $C$. lutea y de manera continua o errática en el resto de las especies evaluadas $(82,4 \%)$ y tal como ha sido observado para el caso del desarrollo vegetativo, B. graveolens, C. parviflora, E. ruizii y L. huasango, no florecen en ese mismo periodo del año; en general la floración abarcó un periodo de 3 a 12 meses con una media de 8,4

Tabla 4. Correlación entre la fluctuación de la precipitación (Pp) temperatura media mensual (TMM) con el numero mensual de especies que mostraron desarrollo vegetativo, floración y fructificación. (* $p>0,05)$

\begin{tabular}{llcc}
\hline & & Pp & TMM \\
\hline \multirow{4}{*}{ Fructificación } & Spearman, $\rho$ (rho) & 0,544 & ${ }^{*} 0,6262$ \\
& Sig. (2-colas) & 0,0673 & 0,0293 \\
& $\mathrm{n}$ & 12 & 12 \\
\hline \multirow{3}{*}{ Floracion } & Spearman, $\rho$ (rho) & 0,5274 & ${ }^{*} 0,5851$ \\
& Sig. (2-colas) & 0,0780 & 0,0456 \\
& $\mathrm{n}$ & 12 & 12 \\
\hline \multirow{3}{*}{ Vegetativo } & Spearman, $\rho$ (rho) & 0,3907 & ${ }^{*} 0,8052$ \\
& Sig. (2-colas) & 0,2092 & 0,0015 \\
& $\mathrm{n}$ & 12 & 12 \\
\hline
\end{tabular}


Tabla 5. Estructura vegetal utilizada y época del año de ocurrencia de las especies que sirven de alimento a la pava aliblanca en la REP Chaparrí, Chongoyape (Lambayeque-Perú) 1 .

\begin{tabular}{|c|c|c|c|c|c|c|c|}
\hline \multirow[b]{2}{*}{$\mathrm{N}^{\circ}$} & \multirow[b]{2}{*}{ Especie } & \multicolumn{5}{|c|}{ Estructura vegetal } & \multirow{2}{*}{$\begin{array}{c}\text { Época del año (floración y } \\
\text { fructificación) }\end{array}$} \\
\hline & & Hoj. & Brot. & Bot. y Fl. & Fr. Ver. & Fr. Mad. & \\
\hline 1 & Acacia macracantha & & $x$ & $x$ & $x$ & $x$ & Todo el año \\
\hline 2 & Acnistus arborescens & & & $x$ & & $x$ & Todo el año \\
\hline 3 & Bursera graveolens & $x$ & & & $x$ & $x$ & Nov-Jun \\
\hline 4 & Cesalpinia paipai & & & $x$ & $x$ & $x$ & May-Mar \\
\hline 5 & Capparis avicennifolia & & $x$ & $x$ & & $x$ & Todo el año \\
\hline 6 & Capparis scabrida & & $x$ & $x$ & & & Todo el año \\
\hline 7 & Carica parviflora & & & $x$ & & $x$ & Oct-Jun \\
\hline 8 & Cercidium praecox & & & $x$ & $x$ & $x$ & Jul-Mar \\
\hline 9 & Cordia lutea & $x$ & $x$ & $x$ & & $x$ & Todo el año \\
\hline 10 & Eriotheca ruizii & & & $x$ & & $x$ & Feb-Dic \\
\hline 11 & Ficus nymphaeifolia & & & & & $x$ & Jul-May \\
\hline 12 & Loxopterigium huasango & & $x$ & $x$ & & & Dic-Jul \\
\hline 13 & Muntingia calabura & $x$ & & $x$ & & $x$ & Todo el año \\
\hline 14 & Pithecellobium excellsum & $x$ & & $x$ & $x$ & $x$ & Jul-May. \\
\hline 15 & Phithecolobium multiflorum & $x$ & & & $x$ & $x$ & Ene-Nov \\
\hline 16 & Prosopis pallida & & $x$ & $x$ & $x$ & $x$ & Jul-Abr \\
\hline 17 & Scutia spicata & & & & & $x$ & Ago-Set/Ene-Mar \\
\hline
\end{tabular}

1 Hoj., hojas; Brot., brotes; Bot. y Fl. Botones y flores; Fr. Ver., fruto verde; Fr. Mad., fruto maduro.

meses. La fructificación, asimismo, ha sido observada apenas en una sola especie durante todo el año $(5,8 \%), A$. arborescens, en tanto que en el resto de especies evaluadas $(94,2 \%)$ ocurre de manera simultánea a la floración o inmediatamente después; en general la fructificación abarcó un periodo de 4 a 12 meses con una media de 8,2 meses.

Se observa una correlación (Spearman) positiva y significativa entre la fluctuación de la temperatura media mensual con el número mensual de especies que mostraron desarrollo vegetativo, floración y fructificación, mas no con la precipitación (Tabla 4, Fig. 2). Es de esperar que la fenología de las especies estudiadas, al igual que otras especies vegetales neotropicales, además de la precipitación y la temperatura estén influenciadas por el fotoperíodo, la evaporación e irradiancia, el suelo, el viento, y factores bióticos como la genética, fisiológica, nutrición, además de las interrelaciones planta-animal y planta-planta (Zárate et al., 2006).

\section{Utilización por la pava aliblanca y en general}

En la tabla 5 se especifica la utilidad de las especies evaluadas en la alimentación de la pava aliblanca. Estos resultados nos indican que 6 especies $(35,3 \%)$ de las 17 evaluadas constituyen la oferta alimenticia de la pava aliblanca durante todo el año, en tanto que de las 11 especies restantes (64,7\%) la oferta alimenticia es de carácter estacional; sin embargo, observamos que durante el año la pava aliblanca siempre dispone de alguna de las especies evaluadas. Con excepción de C. scabrida y L. huasango, la pava aliblanca utilizaría mayormente los frutos maduros, lo que hace un total de 15 especies $(88,2 \%)$, seguido de las flores y botones florales con 13 especies $(76,5 \%)$ y finalmente frutos verdes, brotes y hojas, con alrededor de $40 \%$ para cada caso. Ello nos permite inferir que el riesgo alimenticio para la pava aliblanca estaría relacionado con la ocurrencia de ańos muy secos que impidan el proceso normal de desarrollo vegetativo y desarrollo reproductivo (flores y frutos) así como también la deforestación.

\section{Agradecimiento}

Los autores agradecen a Fernando Angulo Pratolongo, a la Asociación Cracidae, a la Comunidad Campesina Santa Catalina de Chongoyape, a Heinz Plengue, Director de la REP Chaparrí y a Jorge Chanamé Céspedes, de la Universidad Nacional Pedro Ruiz Gallo de Lambayeque, por su invalorable colaboración en los análisis estadísticos.

\section{Literatura citada}

Aguilar, P. 1994. Características faunísticas del norte del Perú. Arnaldoa 2(1): 77-102.

Alencar, J. 1994. Fenología de cinco especies arbóreas tropicais de Sapotaceae correlacionada a variáveis climáticas na Reserva Ducke, Manaus. Manaus-Brasil. Acata Amazonica 24(3/4): 161-182.

Borchert, R. 1995. Phenology and Flowering Periodicity of Neotropical Dry Forest Species: Evidence from Herbarium Collections. Journal Tropical Ecology. Cambridge University Press. USA.

Brack, A. \& C. Mendiola. 2000. Ecología del Perú. PNUD. Asociación Editorial Bruño. Lima, Perú. 495 p.

Bridgewater, S., R.T. Pennington, C.A. Reynel, A. Daza \& T.D. Pennington. 2003. A preliminary floristic and phytogeographic analysis of the woody flora of seasonally dry forests in northern Peru. Candollea 58(1): 129-148.

Croat, T. 1969. Seasonal Flowering Behavior in Central Panama. Ann. Missouri Bot. Gard. 56: 295-307.

De Macedo, H. 1979. Redescubrimiento de la Pava Aliblanca Penelope albipennis Taczanowski 1877. Boletín de Lima Vol. IV. Lima. Pp. 5 - 11.

Díaz, V. 1998. Pava aliblanca (Penelope albipennis): Redescubrimiento, manejo y conservación. Bosques Secos y Desertificación. Memorias del Seminario Internacional. INRENA- Proyecto Algarrobo Perú. Lambayeque, Perú.

Gautier, L. \& R. Spichiger. 1986. Ritmos de reproducción en el estrato arbóreo del Arboretum Jenaro Herrera (provincia de Requena, departamento de Loreto, Perú). Contribución al estudio de la flora y de la vegetación de la Amazonia Peruana. X. Candollea 41 (1): 193-207. 
Gentry, A.H. \& R. Ortiz. 1993. Patrones de Composición Florística en la Amazonía Peruana. Pags. 155-166. En: R. Kalliola, M. Puhakka \& W. Danjoy (eds.). Amazonía Peruana - Vegetación Húmeda en el Llano Subandino. Proyecto Amazonía de la Universidad de Turku, Finlandia y ONERN-Perú, Jyvaskyla.

Leal-Pinedo, J.M. \& Linares-Palomino, R. 2005. Los bosques secos de la Reserva de Biosfera del Noroeste (Perú): Diversidad arbórea y estado de conservación. Caldasia 27(2): 195-211.

Lerner, T. 2003. Etnobotánica de los recursos vegetales de la comunidad "Santa Catalina", microcuenca del río Chancay, distrito de Chongoyape, provincia de Chiclayo, departamento de Lambayeque. Tesis Lic. Biología. Universidad Nacional Agraria La Molina. Perú.

Linares-Palomino, R. \& S.I. Ponce-Álvarez. 2005. Tree community patterns in seasonally dry tropical forests in the Cerros de Amotape Cordillera, Tumbes, Peru. Forest Ecology and Management 209: 261-272.
Ruiz, F. 2000. Evaluación fenológica de diez especies forestales de los bosques productivos de la Comunidad Nativa Santa Mercedes - Río Putumayo; Loreto-Perú. Tesis para optar el título profesional de Ingeniero Forestal. UNAP, IquitosPerú. $128 \mathrm{p}$

Sagástegui, A., M.O. Dillon, I. Sánchez, S. Leiva \& P. Lezama. 1999. Diversidad Florística del Norte del Perú. Tomo I. Trujillo: Edit. Graficart.

Silva, A. \& R. Higaona. 1985. Fenología de las principales especies forrajeras de las Pampas de Olmos. En Centro Internacional de Investigación para el Desarrollo 1991. Investigación sobre Forrajeras Xerófitas y Capricultura (1984 - 1989). Lima, Perú.

Villasana, R. \& A. Súarez. 1997. Estudio fenológico de dieciséis especies forestales presentes en la Reserva Forestal Imataca Estado Bolivar-Venezuela. Revista Forestal Venezolana 41 (1): 13-21.

Zárate, R., Amasifuén, C. \& Flores, M. 2006. Floración y fructificación de plantas leñosas en bosques secos de arena blanca y de suelo arcilloso en la Amazonía Peruana. Rev. per. Biol. 13(1): 095-102. 